\title{
O papel da vigilância entomológica no primeiro foco ativo urbano de Leishmaniose Visceral do município do Rio de Janeiro
}

\author{
The role of entomological surveillance in the first urban active focus of \\ Visceral Leishmaniasis in the city of Rio de Janeiro \\ Cláudio Manuel Rodrigues ${ }^{1}$

\begin{abstract}
RESUMO
Introdução: A leishmaniose Visceral (LV) é uma antropozoonose causada pela Leishmania infantum que avança nos últimos trinta anos a passos largos em sentido ao ambiente urbano. Desde 1977 há registros da doença em áreas periféricas do município do Rio de Janeiro, entretanto em 2011 foi descrita no bairro do Caju por meio do diagnóstico do parasita em cães infectados e da captura de espécimes de seu principal vetor. Objetivo: Este artigo tem por objetivo divulgar o trabalho primário de captura e identificação entomológica destes espécimes que proporcionou ações de vigilância no primeiro foco ativo urbano de LV na cidade do Rio de Janeiro, citado recentemente em diversos estudos, mas ainda não publicizado em todos os seus detalhes. Métodos: As armadilhas luminosas CDC adaptadas foram instaladas de forma setorizada em área circular de $1000 \mathrm{~m}$ de raio a partir do suposto ponto focal, das $17 \mathrm{~h} 00 \mathrm{~m}$ às 07h00m da manhã seguinte, entre junho e setembro de 2011. Resultados: Foram capturados 253 espécimes de Lutzomyia longipalpis, dos quais $63,1 \%$ na área do canil, considerado o foco primário de dispersão dos vetores. Conclusão: Os resultados descritos corroboram com artigos que indicam a urbanização do principal vetor da LV, salientando a importância de estudos mais detalhados sobre o seu ciclo biológico urbano e a necessidade da mudança dos paradigmas da vigilância das leishmanioses no Brasil.
\end{abstract}

Palavras-chave: Leishmaniose visceral; Psychodidae; entomologia; Vigilância Sanitária Ambiental; urbanização.

\begin{abstract}
Introduction: Leishmaniasis, Visceral (LV) is an anthropozoonosis caused by Leishmania infantum that was advanced in the last thirty years in the urban environment. Since 1977 there are records of the disease in peripheral areas of the municipality of Rio de Janeiro, however, in 2011 it was described in the Caju neighborhood by means of the diagnosis of the parasite in infected dogs and the capture of specimens of its main vector. Objective: This article aims to disclose the primary work of entomological capture and identification of these specimens that provided surveillance actions in the first urban active focus of LV in the city of Rio de Janeiro, quoted recently in several studies, but not publicized in all its details. Methods: The adapted CDC light traps were installed in a circular sector of $1000 \mathrm{~m}$ radius from the supposed focal point, from 5:00 p.m. to 7:00 p.m. the following morning, between June and September 2011. Results: A total of 253 specimens of Lutzomyia longipalpis were collected, 63.1\% of which in the kennel area, considered the primary focus of dispersion of the vectors. Conclusion: The results described corroborate articles indicating the urbanization of the main vector of $L V$, stressing the importance of more detailed studies of its urban life cycle and the need of the changing paradigms of leishmaniasis surveillance in Brazil.
\end{abstract}

Keywords: Leishmaniasis, visceral; Psychodidae; entomology; Environmental Health Surveillance; urbanization.

Recebido em: 25/07/2017

Revisado em: 18/10/2017

Aprovado em: 31/10/2017

Autor para correspondência: Cláudio Manuel Rodrigues - Fundação Oswaldo Cruz, Presidência, Centro de Desenvolvimento Tecnológico em Saúde Avenida Brasil, 4036 - sala 814 - Prédio da Expansão - Manguinhos - CEP: 21040-360 - Rio de Janeiro (RJ), Brasil - E-mail: cmrodrigues@cdts.fiocruz.br Conflito de interesses: nada a declarar. 


\section{INTRODUÇÃO}

A Leishmaniose Visceral (LV), uma antropozoonose causada no Brasil pela Leishmania infantum, é transmitida naturalmente pela picada das fêmeas das espécies Lutzomyia longipalpis em 1912 e Lutzomyia cruzi em 1938 ${ }^{1}$. Além de infectar o homem, a L. infantum já foi identificada parasitando outros mamíferos, tais como raposas, marsupiais, roedores e cães, sendo este último considerado o principal reservatório da Leishmaniose Visceral no país ${ }^{2,3}$.

Inicialmente relacionada aos ambientes rural e silvestre, a LV teve nos últimos trinta anos crescente detecção em áreas urbanizadas, fruto dos movimentos antrópicos comuns aos processos desorganizados da urbanização nacional, sendo descritos surtos em diversas cidades brasileiras ${ }^{4-7}$. Em 1977 foi diagnosticado o primeiro caso humano autóctone de LV no Rio de Janeiro, ainda que em área periférica do município, no bairro de Bangu. De lá até 2006 foram notificados 87 casos humanos na doença, oriundos de áreas periurbanas das vertentes do Maciço da Pedra Branca e do Maciço de Gericinó ${ }^{8}$.

Em geral, a doença se apresenta inicialmente em cães para, posteriormente, serem diagnosticados os casos humanos ${ }^{9}$. No caso de se apresentar em cães é a doença denominada como Leishmaniose Visceral Canina (LVC), comum a diversos bairros da zona oeste do Rio de Janeiro, caso de Realengo, Bangu, Senador Camará, Campo Grande e Gericinó ${ }^{10}$. Apesar dos esforços pela gestão da saúde local para o controle de áreas endêmicas para a LVC no Rio de Janeiro, há uma persistência contumaz de cães soropositivos ${ }^{8}$, inclusive com o relato de caso autócne no bairro de Laranjeiras, zona sul da cidade, ainda no ano de $2010^{11}$.

Quando da constatação pelos profissionais veterinários do Instituto Municipal de Medicina Veterinária Jorge Vaitsman (IJV) de casos autóctones de LVC provenientes do Cemitério do Caju ${ }^{12}$, foram realizadas à época capturas sistematizadas para flebotomíneos na localidade por técnicos da vigilância entomológica das leishmanioses da Gerencia de Fatores de Risco Biológico (CVAS/ GFRB) com o objetivo de detectar existência da espécie responsável pela propagação de LV na região ${ }^{13}$.

Este artigo tem por objetivo divulgar o trabalho primário de captura, identificação entomológica e vigilância ambiental no primeiro foco ativo urbano de LV na cidade do Rio de Janeiro, citado recentemente em diversos estudos a respeito de LV e LVC, mas ainda não publicizado em todos os seus detalhes ${ }^{6,11,15,16}$.

\section{MÉTODO}

O presente estudo foi realizado em um raio de, aproximadamente, um quilômetro em torno do foco inicial de LVC localizado em canil na área de serviço do Cemitério de São Francisco Xavier, considerado como o principal do conjunto de necrópoles denominado de Cemitério do Caju.

O bairro do Caju se localiza na zona portuária do Rio de Janeiro, sendo a sua população estimada pelo Censo de $2010 \mathrm{em}$ 20.477 habitantes, que em sua maior parte $(68,3 \%)$ se encontra na faixa de 15 a 64 anos, distribuída em 6957 domicílios e com um rendimento médio mensal de até 4,9 salários-mínimos por responsável por domicílio ${ }^{14}$.

O canil, localizado na área de serviços de manutenção do cemitério, foi adaptado à manutenção dos cães e apresentava estrutura física simples, com condições higiênicas aceitáveis. Segundo consta, a área passava por uma limpeza geral duas vezes por dia. O piso era de concreto com pouco acabamento, assim como os muros formados por placas de concreto e tijolos parcialmente rebocados. Nos cantos dos muros, assim como no próprio piso, foram detectadas diversas aberturas escavadas por roedores, além de pelos e fezes frescas espalhados ao seu redor, caracterizando a manutenção da atividade desses sinantrópicos no foco em questão.

A coleta dos espécimes se realizou entre os meses de junho e setembro do ano 2011, através de armadilhas luminosas de sucção adaptadas do modelo $\mathrm{CDC}^{17}$. As capturas se iniciavam em torno das $17 \mathrm{~h} 00 \mathrm{~min}$ sendo encerradas em torno das $07 \mathrm{~h} 00 \mathrm{~min}$ da manhã seguinte. Foram utilizadas ao todo 37 armadilhas, cuja distribuição obedeceu a um modelo espacial utilizado com base na fisiologia do vetor, que poderia permanecer em atividade no raio de $1000 \mathrm{~m}$ em relação a um provável ponto focal de dispersão ${ }^{18,19}$ (Figura 1).

Os espécimes capturados foram encaminhados ao laboratório de referência, à época no prédio do Instituto Nacional de Endemias Rurais (Departamento de Ciências Biológicas da Escola Nacional de Saúde Pública-Fiocruz), assim como ao Laboratório Central Noel Nutels (LACEN RJ), e identificados de acordo com nomenclatura proposta por Galati ${ }^{20}$.

\section{RESULTADOS}

Nas capturas realizadas ao longo de três meses, foram encontrados 254 flebótomos, sendo a quase totalidade da espécie Lu. Longipalpis, pois somente uma fêmea da espécie $L u$. sallesi foi encontrada na data de 17 de agosto de 2011 em armadilha localizada na área do crematório, não sendo contabilizada para fins estatísticos (Tabela 1).

Dos 253 espécimes considerados para o estudo, 160 (63,2\%) eram machos, dos quais 101 (63,1\%) foram capturados na área do canil, considerado o foco primário de dispersão dos vetores da LVC. Chama atenção que 92 espécimes (36,4\%) foram encontrados em área limítrofe com o Arsenal de Guerra do Rio (AGR), local de grande movimentação de civis e militares, inclusive dispondo de conjunto habitacional constituído por imóveis residenciais geridos pelo Ministério da Defesa. Entretanto nenhum espécime veio a ser coletado no interior da instalação militar durante o período descrito neste estudo.

Um único macho foi capturado no Hospital Nossa Senhora do Socorro na data de 06 de julho de 2011, o que não reduz a importância do achado, visto que a dispersão dos vetores por meios próprios ou através de propagação por ventos comuns ao ambiente litorâneo é factível de ocorrência. Entendendo que essa perspectiva poderia indicar um impacto negativo à saúde de mais de 50 idosos que ali residiam, a Saúde local, através da Divisão de Vigilância em Saúde/ CAP 1.0, suscitou ações integradas à administração do nosocômio em questão. 


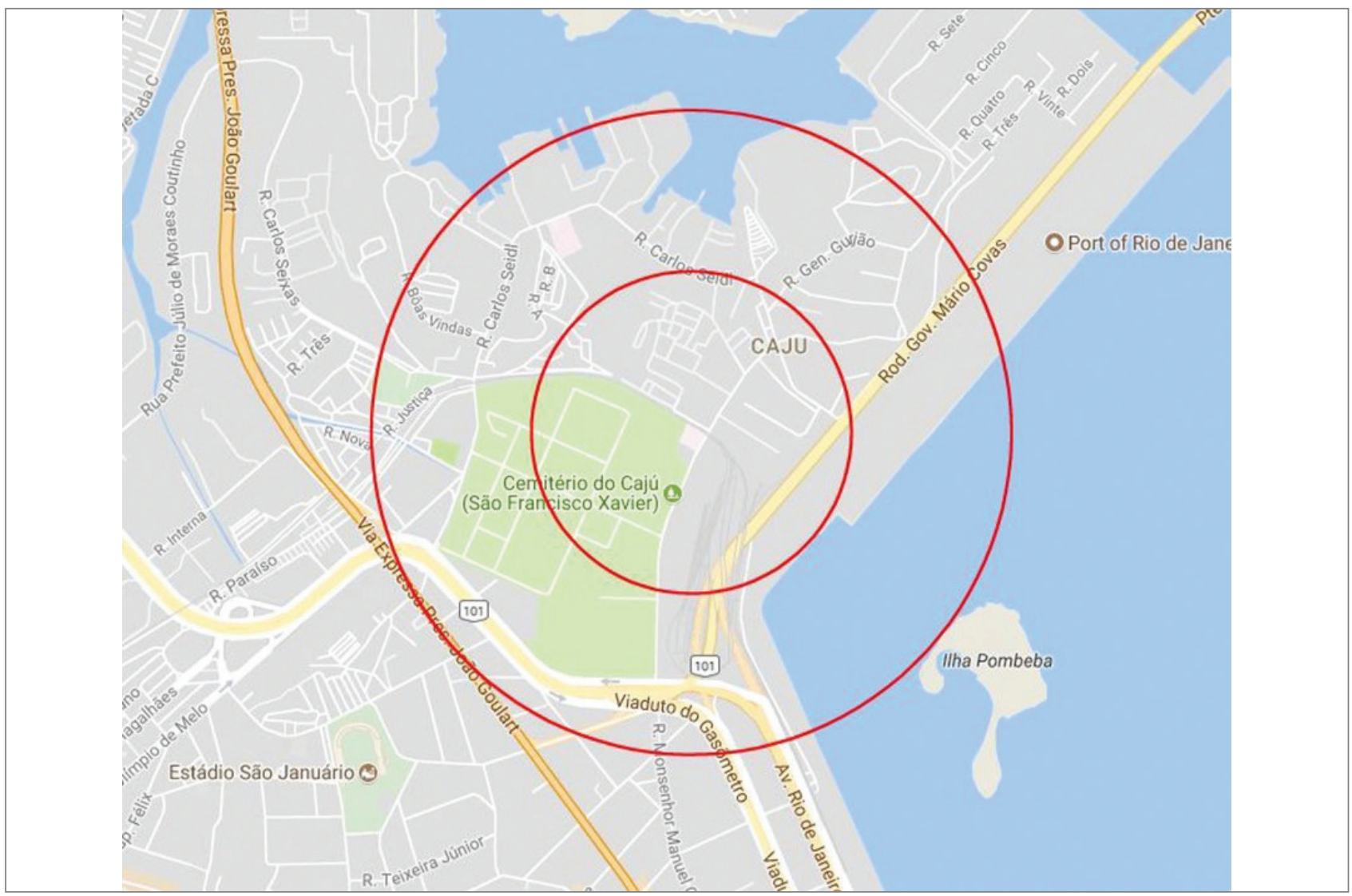

Figura 1: Distribuição espacial de armadilhas luminosas utilizadas na captura dos espécimes de Lutzomyia longipalpis no bairro do Caju, Rio de Janeiro, RJ

Tabela 1: Distribuição dos 253 espécimes de Lutzoyia longipalpis capturados entre junho e setembro de 2011 no bairro do Caju por data, localidade e sexo

\begin{tabular}{|c|c|c|c|c|c|c|c|c|c|c|}
\hline \multirow{2}{*}{ Data } & \multicolumn{2}{|c|}{ Canil } & \multicolumn{2}{|c|}{ HNSS } & \multicolumn{2}{|c|}{$\begin{array}{l}\text { limite } \\
\text { com AGR }\end{array}$} & \multicolumn{2}{|c|}{ Crematório } & \multicolumn{2}{|c|}{ Subtotal } \\
\hline & M & F & M & $\mathbf{F}$ & M & F & M & F & M & $F$ \\
\hline $14 / 06$ & 1 & 3 & 0 & 0 & 0 & 0 & 0 & 0 & 1 & 3 \\
\hline $15 / 06$ & 0 & 0 & 0 & 0 & 0 & 0 & 0 & 0 & 0 & 0 \\
\hline $16 / 06$ & 0 & 0 & 0 & 0 & 0 & 0 & 0 & 0 & 0 & 0 \\
\hline 05/07 & 1 & 4 & 0 & 0 & 0 & 0 & 0 & 0 & 1 & 4 \\
\hline 06/07 & 26 & 6 & 1 & 0 & 0 & 0 & 0 & 0 & 27 & 6 \\
\hline 07/07 & 8 & 2 & 0 & 0 & 0 & 0 & 0 & 0 & 8 & 2 \\
\hline $12 / 07$ & 16 & 3 & 0 & 0 & 0 & 0 & 0 & 0 & 16 & 3 \\
\hline $13 / 07$ & 13 & 5 & 0 & 0 & 0 & 0 & 0 & 0 & 13 & 5 \\
\hline $14 / 07$ & 16 & 9 & 0 & 0 & 0 & 0 & 0 & 0 & 16 & 9 \\
\hline $16 / 08$ & 6 & 1 & 0 & 0 & 4 & 0 & 3 & 2 & 13 & 3 \\
\hline $17 / 08$ & 2 & 2 & 0 & 0 & 0 & 1 & 6 & 1 & 8 & 4 \\
\hline $18 / 08$ & 11 & 7 & 0 & 0 & 1 & 2 & 0 & 0 & 12 & 9 \\
\hline $23 / 08$ & 1 & 0 & 0 & 0 & 14 & 26 & 0 & 0 & 15 & 26 \\
\hline $24 / 08$ & 0 & 0 & 0 & 0 & 8 & 7 & 0 & 0 & 8 & 7 \\
\hline $25 / 08$ & 0 & 0 & 0 & 0 & 9 & 11 & 0 & 0 & 9 & 11 \\
\hline $30 / 08$ & 0 & 0 & 0 & 0 & 2 & 0 & 0 & 0 & 2 & 0 \\
\hline $31 / 08$ & 0 & 0 & 0 & 0 & 1 & 0 & 0 & 0 & 1 & 0 \\
\hline $01 / 09$ & 0 & 0 & 0 & 0 & 3 & 0 & 0 & 0 & 3 & 0 \\
\hline $13 / 09$ & 0 & 0 & 0 & 0 & 0 & 0 & 3 & 0 & 3 & 0 \\
\hline $14 / 09$ & 0 & 0 & 0 & 0 & 2 & 1 & 0 & 0 & 2 & 1 \\
\hline $15 / 09$ & 0 & 0 & 0 & 0 & 0 & 0 & 2 & 0 & 2 & 0 \\
\hline Subtotal & 101 & 42 & 1 & 0 & 44 & 48 & 14 & 3 & 160 & 93 \\
\hline
\end{tabular}

Arsenal de Guerra do Rio: AGR; HNSS: Hospital Nossa Senhora do Socorro; $M=$ macho; $F$ : fêmea

\section{DISCUSSÃO}

O resultado positivo para presença dos vetores da LV obtido nas coletas realizadas em um raio de até $500 \mathrm{~m}$ da área do canil já seria o suficiente para inferir estes locais como mantenedores de flebotomíneos, entretanto serão necessários estudos mais criteriosos com o intuito de qualificar a participação dos animais (cães, roedores, entre outros) na manutenção do ciclo biológico de $L u$. Longipalpis e, se possível, observar a presença de formas imaturas possivelmente dispersas na região adstrita ao cemitério.

Associado a este fato, a questão de 25 cães terem sido diagnosticados como positivos para LVC levou a classificação do local como o primeiro foco ativo urbano para a doença na cidade do Rio de Janeiro ${ }^{14}$, exigindo um planejamento de ações que integrasse distintas áreas da saúde humana, animal e ambiental em uma perspectiva de Saúde Única ${ }^{12,13,21}$.

Portanto, conclui-se que a presença de Lu. longipalpis em consolidada área urbana do município do Rio de Janeiro, à época ainda sem notificação de caso humano autócne ${ }^{15}$, reforça a tendência de adaptação desta espécie aos ambientes antropizados, como já descrito por diversos estudiosos ${ }^{1,5,6,8}$, exigindo das autoridades sanitárias um pensar novo para lidar com este paradigma que se apresenta na análise ecoepidemiológica da LV em conglomerados urbanos brasileiros. 


\section{REFERÊNCIAS}

1. Lainson R, RangelEF. Lutzomyialongipal pis and the eco-epidemiology of American visceral leishmaniasis, with particular reference to Brazil: a review. Mem Inst Oswaldo Cruz. 2005;100(8):811-27. http://dx.doi.org/10.1590/S0074-02762005000800001

2. Brasil. Ministério da Saúde. Secretaria de Vigilância em Saúde. Guia de vigilância em saúde: volume único. Brasília: Ministério da Saúde; 2014.

3. Deane L. Leishmaniose visceral no Brasil: estudos sobre reservatórios e transmissores realizados no Estado do Ceará. Rio de Janeiro: Serviço Nacional de Educação Sanitária; 1956.

4. Monteiro EM, Silva JC, Costa RT, Costa DC, Barata RA, Paula EV, et al. Visceral leishmaniasis: a study on phlebotomine sand flies and canine infection in Montes Claros, State of Minas Gerais. Rev Soc Bras Med Trop 2005;38(2):147-52. http://dx.doi.org/10.1590/S0037-86822005000200004

5. Werneck GL. Fórum: Expansão geográfica e urbanização da leishmaniose visceral no Brasil. Introdução. Cad Saúde Pública. 2008;24(12):2937-40.

http://dx.doi.org/10.1590/S0102-311X2008001200023

6. Brazil RP. The dispersion of Lutzomyia longipalpis in urban areas. Rev Soc Bras Med Trop. 2013;46(3):263-4. http://dx.doi.org/10.1590/0037-8682-0101-2013

7. Silva DA, Madeira MF, Figueiredo FB. Geographical expansion of canine visceral leishmaniasis in Rio de Janeiro State, Brazil. Rev Inst Med Trop São Paulo. 2015; 57(5):435-8. http://dx.doi.org/10.1590/S0036-46652015000500012

8. Marzochi MCA, Fagundes A, Andrade MV, Souza MB, Madeira MF, Mouta-Confort E, et al. Visceral leishmaniasis in Rio de Janeiro, Brazil: eco-epidemiological aspects and control. Rev Soc Bras Med Trop. 2009;42(5):570-80. http://dx.doi.org/10.1590/S0037-86822009000500017

9. Bevilacqua PD, Paixão HH, Modena CM, Castro MCPS. Urbanização da leishmaniose visceral em Belo Horizonte. Arq Bras Med Vet Zootec. 2001;53(1):1-8. http://dx.doi.org/10.1590/S0102-09352001000100001

10. Madeira MF, Schubach AO, Schubach TM, Pereira SA, Figueiredo FB, Baptista C, et al. Post mortem parasitological evaluation of dogs seroreactive for Leishmania from Rio de Janeiro, Brazil. Vet Parasitol. 2006;138(3-4):366-70. http://dx.doi.org/10.1016/j.vetpar.2006.01.059

11. Figueiredo FB, Barbosa Filho CJ, Schubach EY, Pereira SA, Nascimento LD, Madeira MF. Relato de caso autóctone de leishmaniose visceral canina na zona sul do município do Rio de Janeiro. Rev Soc Bras Med Trop. 2010;43(1):98-9. http://dx.doi.org/10.1590/S0037-86822010000100022
12. Prefeitura do Rio de Janeiro. Secretaria Municipal de Saúde e Defesa Civil. Subsecretaria de Vigilância, Fiscalização Sanitária e Controle de Zoonoses. Superintendência de Vigilância e Fiscalização Sanitária em Zoonoses. Nota técnica n. ${ }^{\circ} 01$ /2011/s/ SUBVISA /SVFSZ: Orientações sobre vigilância, prevenção e controle da Leishmaniose Visceral (LV) em caninos, no município do Rio de Janeiro (MRJ), em virtude da confirmação de transmissão canina da doença, no bairro do Caju - Área Programática. Disponível em: http://www.crmvrj.org.br/nota\%20 tecnica\%20Leishmaniose_SUBVISA.pdf. Acesso em: 17 jul. 2017.

13. Prefeitura do Rio de Janeiro. Secretaria Municipal de Saúde. Superintendência de Vigilância em Saúde. A Vigilância em Saúde na Cidade do Rio de Janeiro. Rio de Janeiro: SMS; 2014. Disponível em: http://www.rio.rj.gov.br/dlstatic/10112/5003182/4128740/ AVigilanciaemSaudenaCidadedoRiodeJaneiroed.2014.pdf. Acesso em: 19 jul. 2017.

14. Prefeitura do Rio de Janeiro. Instituto Pereira Passos (IPP). Bairros cariocas. Disponível em: http://portalgeo.rio.rj.gov.br/ bairroscariocas/index_bairro.htm. Acesso em: 17 jul. 2017.

15. Silva GAR, Boechat TO, Ferry FRA, Pinto JFC, Azevedo MCVM, Carvalho RS, et al. First case of autochthonous human visceral leishmaniasis in the urban center of Rio de Janeiro: case report. Rev Inst Med Trop Sao Paulo 2014; 56(1):81-4. http://dx.doi.org/10.1590/S0036-46652014000100013

16. Marzochi MCA. Visceral leishmaniasis in Southern Rio de Janeiro State and the risk of propagation to São Paulo State, Brazil. Rev Soc Bras Med Trop 2016;49(2):147-9.

http://dx.doi.org/10.1590/0037-8682-0442-2015

17. Falcão AR. Um novo modelo de armadilha luminosa de sucção para pequenos insetos. Mem Inst Oswaldo Cruz. 1981;76(3):303-5. http://dx.doi.org/10.1590/S0074-02761981000300009

18. Pinheiro MVB, Brazil RM, Maspero RC, Araujo SQ, Rodrigues CM. Distribuição espacial da Lutzomyia longipalpis em área urbana do município do Rio de Janeiro. Acta Scientiae Veter. 2012;40(Supl 2):s66.

19. Aparício C, Bittencourt MD. Análise espacial da leishmaniose tegumentar americana. Anais do XI Simpósio Brasileiro de Sensoriamento Remoto. 2003; 05-10 abr; Belo Horizonte, Brasil. INPE; 2003; p.1247-54.

20. Galati EAB. Classificação de Phlebotominae. In: Rangel EF, Lainson R. Flebotomíneos do Brasil. Rio de Janeiro: Fiocruz; 2003; p. 23-51.

21. Day MJ. One health: the importance of companion animal vectorborne diseases. Parasit Vectors. 2011:4:49. https://dx.doi.org/10.1186/1756-3305-4-49 